\title{
Gene expression profiles reveal key pathways and genes associated with neuropathic pain in patients with spinal cord injury
}

\author{
XIJING HE ${ }^{1}$, LIYING FAN ${ }^{1}$, ZHONGHENG WU ${ }^{2}$, JIAXUAN $\mathrm{HE}^{3}$ and BIN CHENG ${ }^{1}$ \\ Departments of ${ }^{1}$ Orthopedics, ${ }^{2}$ Rehabilitation and ${ }^{3}$ Anesthesiology, \\ The Second Affiliated Hospital of Xi'an Jiaotong University, Xi'an, Shaanxi 710004, P.R. China
}

Received December 30, 2015; Accepted December 8, 2016

DOI: $10.3892 / \mathrm{mmr} .2017 .6231$

\begin{abstract}
Previous gene expression profiling studies of neuropathic pain (NP) following spinal cord injury (SCI) have predominantly been performed in animal models. The present study aimed to investigate gene alterations in patients with spinal cord injury and to further examine the mechanisms underlying NP following SCI. The GSE69901 gene expression profile was downloaded from the public Gene Expression Omnibus database. Samples of peripheral blood mononuclear cells (PBMCs) derived from 12 patients with intractable NP and 13 control patients without pain were analyzed to identify the differentially expressed genes (DEGs), followed by functional enrichment analysis and protein-protein interaction (PPI) network construction. In addition, a transcriptional regulation network was constructed and functional gene clustering was performed. A total of 70 upregulated and 61 downregulated DEGs were identified in the PBMC samples from patients with NP. The upregulated and downregulated genes were significantly involved in different Gene Ontology terms and pathways, including focal adhesion, $\mathrm{T}$ cell receptor signaling pathway and mitochondrial function. Glycogen synthase kinase $3 \beta(G S K 3 B)$ was identified as a hub protein in the PPI network. In addition, ornithine decarboxylase 1 $(O D C 1)$ and ornithine aminotransferase $(O A T)$ were regulated by additional transcription factors in the regulation network. $G S K 3 B, O A T$ and $O D C 1$ were significantly enriched in two functional gene clusters, the function of mitochondrial membrane and DNA binding. Focal adhesion and the T cell receptor signaling pathway may be significantly linked with NP, and $G S K 3 B, O A T$ and $O D C 1$ may be potential targets for the treatment of NP.
\end{abstract}

Correspondence to: Dr Xijing He, Department of Orthopedics, Second Affiliated Hospital of Xi'an Jiaotong University, 157 Xiwu Road, Xincheng, Xi'an, Shaanxi 710004, P.R. China

E-mail: xijinghee@163.com

Key words: spinal cord injury, neuropathic pain, functional enrichment analysis, protein-protein interaction network, gene clustering

\section{Introduction}

Spinal cord injury (SCI) is an ever-increasing challenge and a devastating neurologic event affecting patients and their families (1). A myriad of neurologic and medical sequelae can occur subsequent to the original insult, including the long-term loss of sensory and motor functions, amongst other complications (1). Chronic pain is an important problem following SCI and is a major impediment to effective rehabilitation (2). It has been shown that chronic pain following SCI has a high prevalence of at least $80 \%$, which in at least $40 \%$ of individuals manifests as persistent neuropathic pain (NP) (3).

$\mathrm{NP}$ can be categorized as peripheral and central pain, and is often described as paroxysmal, stabbing, burning, pulsing, electric shock-like, pricking or tingling, and a spontaneous or evoked unpleasant abnormal sensation (4). Current treatments use a variety of surgical, pharmacological, physical and psychological strategies for the management of NP following SCI (2). However, evidence of effective therapeutic approaches in use remains to be fully elucidated. Therefore, an improved understanding of the mechanisms underlying NP in patients with SCI and the identification of effective treatment strategies are required.

In previous years, the identification of specific molecular alterationsinvolved inNPsyndromeshas becomeamajorpriority in investigations of SCI (5). Several biological alterations have been implicated in the mechanisms of NP, including cellular interactions, ion channel expression, extracellular proteins and epigenetic effects (6). For example, N-type voltage-dependent $\mathrm{Ca}^{2+}$ channels, which are expressed in non-excitable microglial cells in mice, have been demonstrated to contribute to the pathophysiology of NP (7). In addition, Chen et al (8) demonstrated that astrocytic connexin-43 enhances spinal cord synaptic transmission and maintains late-phase NP in mice via the release of chemokines. Nesic et al (9) performed DNA microarray analysis and showed that a number of genes with increased expression were significantly associated with astrocytic activation and inflammation in the spinal cords of rats, which developed central NP. Vicuña et al (10) revealed that the serine protease inhibitor, serpin $\mathrm{A} 3 \mathrm{~N}$ can attenuate $\mathrm{NP}$ by inhibiting $\mathrm{T}$ cell-derived leukocyte elastase in mice, and demonstrated crosstalk between $\mathrm{T}$ cells and neurons in the modulation of NP. However, investigations of NP following SCI have predominantly been performed in animal models 
and the exact molecular mechanisms of persistent NP remain to be elucidated.

Microarray data provide a global assessment of gene expression signatures, which may provide insights into the pathophysiology of disease (11). Previous gene expression profiling studies of NP following SCI have been performed predominantly in animal models $(12,13)$. However, the gene expression profiling of NP in human whole blood has not been reported. In the present study, the microarray data of GSE69901 was downloaded from the publicly available Gene Expression Omnibus (GEO) database and analyzed. Differentially expressed genes (DEGs) were screened in the peripheral blood mononuclear cells (PBMCs) of samples from patients with SCI and intractable NP, and compared with those from patients with SCI without pain. This was followed by functional enrichment analysis and construction of a protein-protein interaction (PPI) network. A transcriptional regulation network was also constructed and functional gene clustering was performed. The aim of the present study was to further investigate the molecular mechanisms underlying NP following SCI, and to identify additional potential pathways and genes associated with the pathogenesis of NP.

\section{Materials and methods}

Microarray data. The GEO (http://www.ncbi.nlm.nih.gov/geo/) is an international public repository, which archives and freely distributes high-throughput microarray and next-generation sequencing functional genomic data deposited by the scientific community (14). In addition to serving as a public archive, the GEO database provides available tools to assist users in identifying, analyzing and visualizing data associated with their specific interests (14). In the present study, the GSE69901 microarray data, deposited by Adigüzel et al on 15th June 2015, was retrieved from the publicly available GEO database (https://www. ncbi.nlm.nih.gov/geo/query/acc.cgi?acc=GSE69901). As shown in the description of the GSE69901 series in the GEO database, the PBMCs were collected from whole blood samples from 12 patients with intractable NP and 13 patients in the control group (without pain). All patients had complete SCI with a level of injury above T5. Data were generated using the platform of the GPL15207 (PrimeView) Affymetrix Human Gene Expression Array. In the present study, the 25 samples were used for the subsequent analysis, comprising the 12 PBMC samples from patients with NP and 13 PBMC samples from patients without pain.

Data preprocessing and differential expression analysis. The raw data (Series Matrix files) were downloaded. According to the annotation information on the GPL15207 platform, the probe symbols were transformed into gene symbols. Gene expression values were averaged using the aggregate function in $\mathrm{R}$ (version 3.3.1, https://www.r-project. org/) when multiple probe sets mapped to a same gene symbol. Missing values of probes were imputed using the k-nearest-neighbor algorithm (15) present in the input package (16) in R. In addition, quartile data normalization was performed using the Bioconductor preprocessCore package (version 1.28.0., http://bioconductor.org/packages/release/
bioc/html/preprocessCore.htmlurisimplehttp://bioconductor. org/packages/release/bioc/html/preprocessCore.html) (17).

A $t$-test in the limma package (version 3.22.7, http://www .bioconductor.org/packages/3.0/bioc/html/limma.html) was performed to identify DEGs in the specimens from the patients with NP, compared with the controls. An absolute value of $\log _{2}$-fold change $\left(\log _{2} \mathrm{FC}\right)>1$ and adjusted $\mathrm{P}$-value of $<0.05$ were used to determine significant differential expression.

Functional enrichment analysis. TargetMine is an integrated database enabling complicated searches, which are difficult to perform using existing comparable tools and assists in efficient target prioritization (18). In order to analyze the identified upregulated and downregulated genes at functional levels, Gene Ontology (GO) and Kyoto Encyclopedia of Genes and Genomes (KEGG) pathway enrichment analyses were performed using TargetMine (http://targetmine.nibio.go.jp/). GO terms or pathways with a P-value $<0.05$ were considered to be significantly enriched.

PPI network construction. The online database resource Search Tool for the Retrieval of Interacting Genes (STRING) provides uniquely comprehensive coverage, and access to predicted and experimental interaction information (19). Interactions in the STRING database are provided with a confidence score (19). In the present study, application of the STRING database (http://string-db.org/) was used to predict PPIs based on a confidence score $>0.4$ and other default parameters. The PPI network was visualized using Cytoscape (version 2.8.2, http://www.cytoscape.org/) (20).

Transcriptional regulation network construction. The Human Transcriptional Regulation Interactions database (HTRIdb) is an open-access database (http://www.lbbc.ibb. unesp.br/htri) from which human experimentally validated interactions among transcription factors (TFs) and their corresponding target genes can be extracted. These can be used to construct transcriptional regulation interaction networks, enabling detailed understanding of the regulation of biological processes (21). In the present study, the TFs involved in regulating all DEGs were screened based on the data derived from the HTRIdb. A transcriptional regulation network was subsequently constructed and visualized in Cytoscape (20).

Gene clustering. The Gene Cluster with Literature Profile (GenCLip) (http://www.genclip.com/) is a literature mining tool for functional clustering of a list of genes with keywords, GO terms or pathways using the average linkage hierarchical clustering algorithm (22). In the present study, all DEGs were analyzed using GenCLip software (version 2.0, http://ci.smu. edu.cn) to identify functional gene clusters.

\section{Results}

Data preprocessing and identification of DEGs. Following data preprocessing, 19,419 genes were mapped to the probes. The gene expression profiles prior to and following normalization are shown in Fig. 1A. The results revealed that, following normalization, the median probe intensity was similar across 


\section{A}

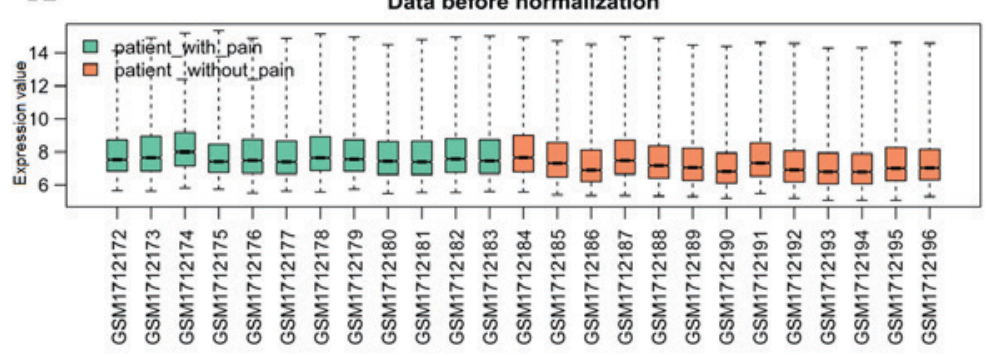

Data after normailization

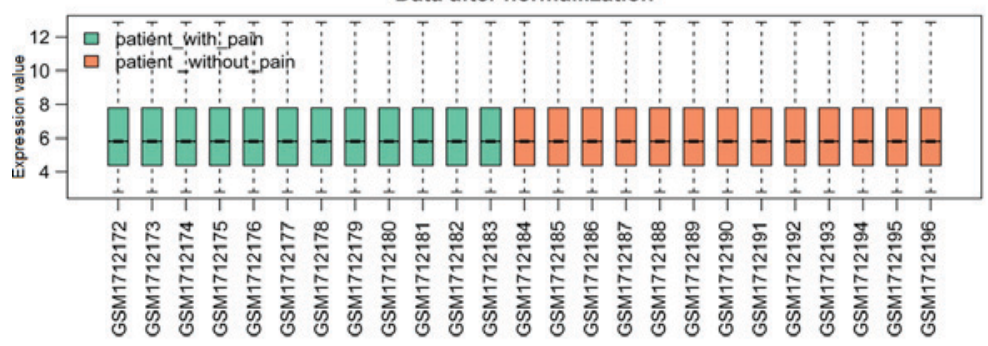

B

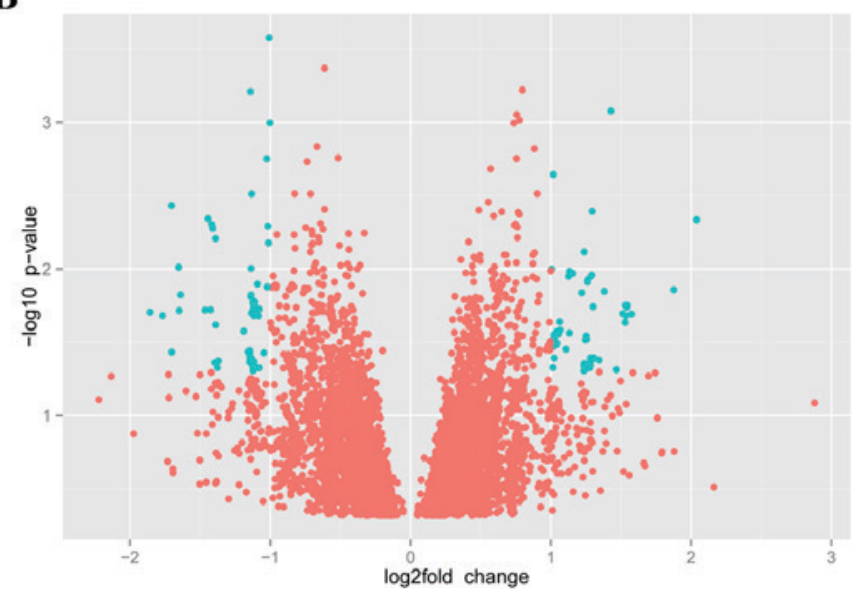

Figure 1. Data preprocessing and screening of DEGs. (A) Normalized expressed value data. The box in the black line indicates the median of each set of data, which determines the degree of standardization of data through its distribution. Following normalization, the black line in the box is almost in the same straight line, indicating a good degree of standardization. (B) Volcano plot of the log 10 false discovery rate against the log (fold-change) of each gene. Green indicates the identified DEGs. Red represents the gene without differential expression. DEGs, differentially expressed genes.

all conditions, which reflects the consistency of the technical quality in the data.

In the original analysis by Adıgüzel et al, only 16 DEGs, including nine upregulated and seven downregulated genes, were obtained with a cut-off degree of a 4-fold change. However, the present study identified a total of 131 DEGs in the PBMC samples from patients with intractable NP, compared with controls under the specific thresholds of $\mid \log _{2} \mathrm{FCl}>1$ and adjusted $\mathrm{P}$-value $<0.05$, including 70 upregulated and 61 downregulated genes. A volcano plot was applied to visualize the genes identified (Fig. 1B). In addition, a heat map of gene expression values was constructed (Fig. 2). The color patterns in the heat map indicate the variability of gene expression between the groups of SCI with NP and without NP.

GO and pathway enrichment analysis. The upregulated and downregulated genes were enriched in different GO terms and pathways. The results showed that the upregulated genes were enriched in categories of 39 biological processes (BPs), four molecular functions (MFs), four cellular components (CCs) and four KEGG pathways. By contrast, the downregulated genes were enriched in GO terms of $15 \mathrm{BPs}$, one $\mathrm{CC}$, nine MFs and nine KEGG pathways. The top five GO terms in each category and the results of the KEGG pathway enrichment analysis are shown in Tables I and II, respectively.

The results revealed that the upregulated genes were predominantly involved in GO terms associated with platelet activation, cell activation, blood coagulation and mitochondrial function. The upregulated genes were also enriched in four pathways, which included focal adhesion and the $\mathrm{T}$ cell receptor signaling pathway. The downregulated genes were involved with cytosolic calcium ion transport and the positive regulation of phospholipase activity. Additionally, the downregulated genes were involved in pathways, including fat digestion and absorption, pancreatic secretion and vascular smooth muscle contraction.

PPI network analysis. As shown in Fig. 3, the constructed PPI network contained 39 nodes and 29 interactions, consisting of 23 upregulated genes and 16 downregulated genes. The results 


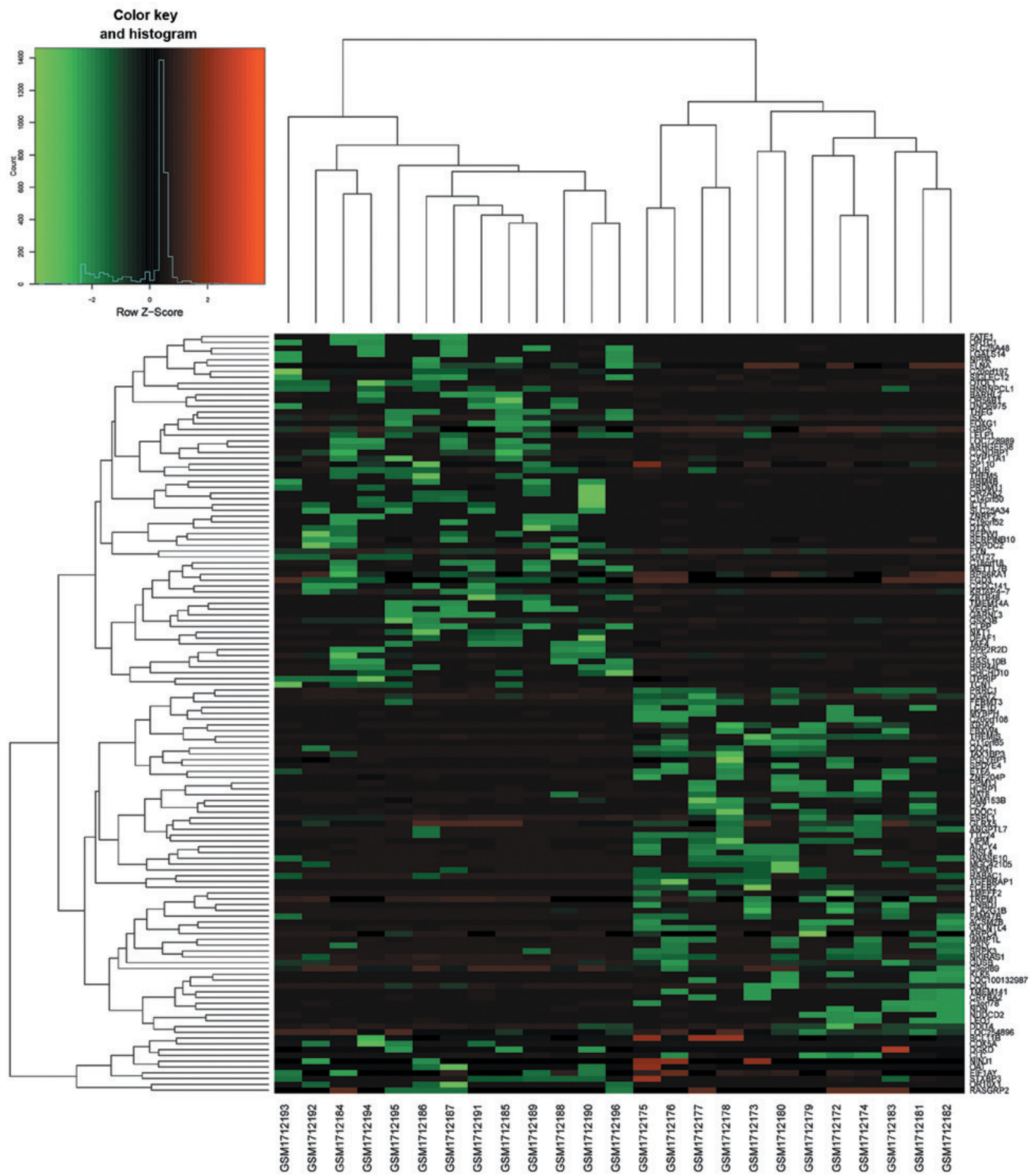

Figure 2. Heat maps of genes, which were significantly differentially expressed in peripheral blood mononuclear cell samples from patients with pain. Red or green colors indicate either higher or lower expression levels of the differentially expressed genes.

revealed that the PPI network had a loose structure. However, glycogen synthase kinase $3 \beta(G S K 3 B)$ and RAS-like, family 10, member B (RASL1OB) had higher node degrees.

Transcriptional regulation network construction and functional gene clustering analysis. The transcriptional regulation network was constructed (Fig. 4). The results revealed that a total of 34 TFs regulated 101 DEGs in the regulation network, comprising 322 regulation pairs. In addition, TFs, including androgen receptor, GATA-binding protein 2 and $\mathrm{Y}$ box-binding protein 1, were found to regulate a higher number of DEGs. Ornithine decarboxylase 1 $(O D C 1)$ (downregulated), ornithine aminotransferase $(O A T)$ (upregulated), diacylglycerol kinase $\delta 130 \mathrm{kDa}(D G K D$; upregulated) were identified to be regulated by a higher number of TFs. It was also found that $O D C 1$ interacted with $O A T$ in the PPI network.

The present study also analyzed the potential functions of the identified DEGs using the GeneCLip text-mining program. The results showed that the DEGs were significantly involved with two functional gene clusters, namely the function of mitochondrial membrane and DNA binding (Table III). In addition, 
Table I. Top five GO terms in each category enriched by upregulated and downregulated genes.

\begin{tabular}{|c|c|c|c|c|c|c|}
\hline Gene change & Ontology & ID & Pathway & P-value & $\mathrm{n}$ & Genes \\
\hline \multirow[t]{11}{*}{ Upregulated } & $\mathrm{BP}$ & GO:0030168 & Platelet activation & $5.16 \mathrm{E}-05$ & 6 & $\begin{array}{l}\text { DGKD, FLNA, FYN, } \\
\text { RASGRP2, STXBP3, VEGFC }\end{array}$ \\
\hline & $\mathrm{BP}$ & GO:0001775 & Cell activation & 0.002258 & 7 & $\begin{array}{l}\text { DGKD, FLNA, FYN, } \\
\text { RASGRP2, RPS6KA1, } \\
\text { STXBP3, VEGFC }\end{array}$ \\
\hline & $\mathrm{BP}$ & GO:0007596 & Blood coagulation & 0.003756 & 6 & $\begin{array}{l}\text { DGKD, FLNA, FYN, } \\
\text { RASGRP2, STXBP3, VEGFC }\end{array}$ \\
\hline & $\mathrm{BP}$ & GO:0050817 & Coagulation & 0.003864 & 6 & $\begin{array}{l}\text { DGKD, FLNA, FYN, } \\
\text { RASGRP2, STXBP3, VEGFC }\end{array}$ \\
\hline & $\mathrm{BP}$ & GO:0007599 & Hemostasis & 0.0039 & 6 & $\begin{array}{l}\text { DGKD, FLNA, FYN, } \\
\text { RASGRP2, STXBP3, VEGFC }\end{array}$ \\
\hline & $\mathrm{CC}$ & GO:0005759 & Mitochondrial matrix & 0.001476 & 5 & $\begin{array}{l}\text { CLPP, CYP11A1, ICT1, } \\
\text { OAT, THEM5 }\end{array}$ \\
\hline & $\mathrm{CC}$ & GO:0044429 & Mitochondrial part & 0.002033 & 7 & $\begin{array}{l}\text { CHCHD10, CLPP, COX5A, } \\
\text { CYP11A1, ICT1, OAT, THEM5 }\end{array}$ \\
\hline & $\mathrm{CC}$ & GO:0005739 & Mitochondrion & 0.008474 & 8 & $\begin{array}{l}\text { CHCHD10, CLPP, COX5A, } \\
\text { CYP11A1, ICT1, MPC1, OAT, } \\
\text { THEM5 }\end{array}$ \\
\hline & $\mathrm{CC}$ & GO:0031091 & Platelet $\alpha$ granule & 0.013661 & 2 & STXBP3, VEGFC \\
\hline & MF & GO:0019992 & Diacylglycerol binding & $2.45 \mathrm{E}-04$ & 2 & DGKD, RASGRP2 \\
\hline & MF & GO:0001948 & Glycoprotein binding & 0.020786 & 2 & FLNA, FYN \\
\hline \multirow[t]{11}{*}{ Downregulated } & BP & GO:0050830 & $\begin{array}{l}\text { Defense response to } \\
\text { Gram-positive bacterium }\end{array}$ & 0.006167 & 2 & PGLYRP1, PLA2G1B \\
\hline & $\mathrm{BP}$ & GO:0010518 & $\begin{array}{l}\text { Positive regulation } \\
\text { of phospholipase activity }\end{array}$ & 0.0173 & 2 & ADCY4, PLA2G1B \\
\hline & $\mathrm{BP}$ & GO:0060401 & $\begin{array}{l}\text { Cytosolic calcium } \\
\text { ion transport }\end{array}$ & 0.020834 & 2 & PLA2G1B, TRPM1 \\
\hline & $\mathrm{BP}$ & GO:0060402 & $\begin{array}{l}\text { Calcium ion transport } \\
\text { into cytosol }\end{array}$ & 0.020834 & 2 & PLA2G1B, TRPM1 \\
\hline & $\mathrm{BP}$ & GO:0010517 & $\begin{array}{l}\text { Regulation of } \\
\text { phospholipase activity }\end{array}$ & 0.021295 & 2 & ADCY4, PLA2G1B \\
\hline & $\mathrm{CC}$ & GO:0015629 & Actin cytoskeleton & 0.038255 & 3 & ARPC4, FERMT3, TAX1BP3 \\
\hline & MF & GO:0005178 & Integrin binding & 0.015317 & 2 & FCER2, FERMT3 \\
\hline & $\mathrm{MF}$ & GO:0016829 & Lyase activity & 0.025162 & 2 & ADCY4, ODC1 \\
\hline & MF & GO:0004175 & Endopeptidase activity & 0.028922 & 3 & CLC, ESPL1, KLK5 \\
\hline & MF & GO:0004197 & $\begin{array}{l}\text { Cysteine-type } \\
\text { endopeptidase activity }\end{array}$ & 0.029558 & 2 & CLC, ESPL1 \\
\hline & MF & GO:0016747 & $\begin{array}{l}\text { Transferase activity, } \\
\text { transferring acyl groups } \\
\text { other than amino-acyl groups }\end{array}$ & 0.033704 & 2 & DGAT2, NAT8 \\
\hline
\end{tabular}

GO, Gene Ontology; MF, molecular function; BP, biological process; CC, cellular component.

the $G S K 3 B, O A T$ and $O D C 1$ DEGs were clearly clustered into the subgroup associated with the function of mitochondrial membrane. As shown in Table III, the GSK3B and ODC1 DEGs were also closely associated with DNA binding.

\section{Discussion}

NP is a common problem following SCI and is usually difficult to treat (23). In the present study, gene expression profiling was performed to further investigate the molecular mechanisms underlying NP in patients with SCI. As a result, a total of 70 upregulated and 61 downregulated genes were identified in the PBMC samples from patients with intractable NP, compared with the controls. The upregulated genes and downregulated genes were significantly involved in different GO terms and pathways, including focal adhesion, the $\mathrm{T}$ cell receptor signaling pathway and mitochondrial function. $G S K 3 B$ was identified as a hub protein in the PPI network. In 
Table II. Enriched pathways of the upregulated and downregulated genes.

\begin{tabular}{|c|c|c|c|c|c|}
\hline Gene change & ID & Pathway & P-value & $\mathrm{n}$ & Genes \\
\hline \multirow[t]{4}{*}{ Upregulated } & hsa04510 & Focal adhesion & 0.005135 & 4 & FLNA, FYN, GSK3B, VEGFC \\
\hline & hsa03015 & mRNA surveillance pathway & 0.039026 & 2 & NXT1, PPP2R2D \\
\hline & hsa04740 & Olfactory transduction & 0.048956 & 4 & OR10X1, OR1C1, OR2AK2, OR56B1 \\
\hline & hsa04660 & T cell receptor signaling pathway & 0.049678 & 2 & FYN, GSK3B \\
\hline \multirow[t]{9}{*}{ Downregulated } & hsa04975 & Fat digestion and absorption & 0.00297 & 2 & DGAT2, PLA2G1B \\
\hline & hsa04972 & Pancreatic secretion & 0.015504 & 2 & ADCY4, PLA2G1B \\
\hline & hsa04114 & Oocyte meiosis & 0.021098 & 2 & ADCY4, ESPL1 \\
\hline & hsa04270 & Vascular smooth muscle contraction & 0.023984 & 2 & ADCY4, PLA2G1B \\
\hline & hsa01100 & Metabolic pathways & 0.024489 & 6 & $\begin{array}{l}\text { ACSM2B, DGAT2, GALNT18, GUSB, } \\
\text { ODC1, PLA2G1B }\end{array}$ \\
\hline & hsa04611 & Platelet activation & 0.027808 & 2 & ADCY4, FERMT3 \\
\hline & hsa00531 & Glycosaminoglycan degradation & 0.037737 & 1 & GUSB \\
\hline & hsa03060 & Protein export & 0.045511 & 1 & IMMP1L \\
\hline & hsa00592 & $\alpha$-linolenic acid metabolism & 0.049376 & 1 & PLA2G1B \\
\hline
\end{tabular}
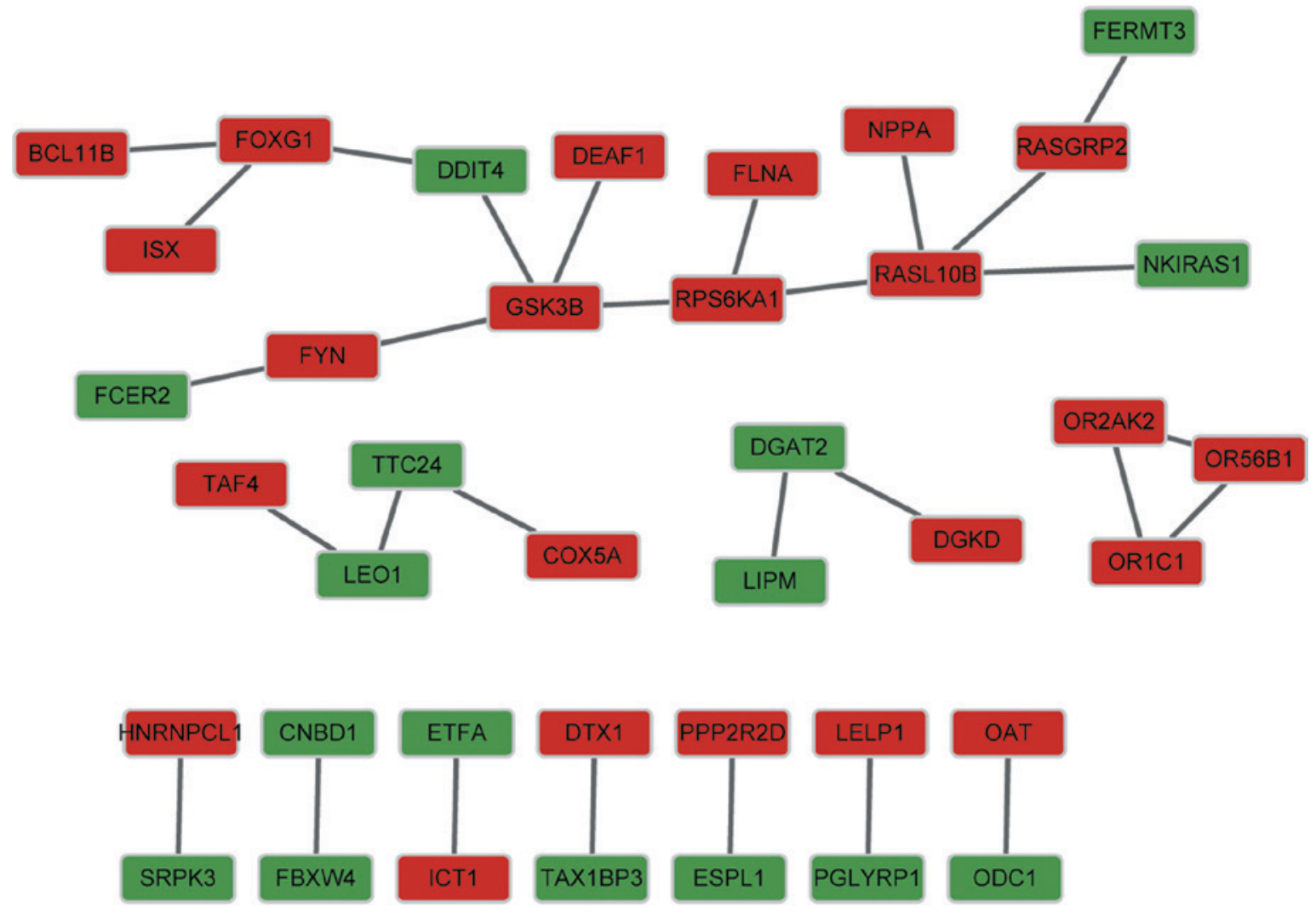

Figure 3. Protein-protein interaction network of differentially expressed genes. The red nodes represent upregulated genes. The green nodes represent downregulated genes.

addition, $O D C 1, D G K D$ and $O A T$ were regulated by a higher number of TFs in the transcriptional regulation network. $G S K 3 B, O A T$ and $O D C 1$ were also significantly enriched in two functional gene clusters, namely the functions of mitochondrial membrane and DNA binding.

In the present study, a total of 131 DEGs (70 upregulated and 61 downregulated) were identified in the PBMC samples from patients with SCI and NP, compared with controls under the specific thresholds. However, in the original analysis by Adigüzel et al, only 16 DEGs (nine upregulated and seven downregulated) were obtained with a cut-off degree of a 4-fold change. Thus, the results of the present study showed that distinct genetic features were identified in PBMC samples using different screening methods with different thresholds.

Previous evidence has led to an increased awareness of the contribution of immune and inflammatory systems to NP, 
Table III. Results of functional gene clustering analysis.

Function of cluster

Genes

P-value

Function of mitochondrial membrane

COX5A, CYP11A1, GSK3B, IMMP1L, MPC1, OAT,

0.001229

DNA binding

ODC1, ROM1, SLC25A34, SLC25A48, TMEM14A

0.048263

DDIT4, DEAF1, DTX1, FCER2, FOXG1, GSK3B,

GUSB, LDOC1, LEO1, NDN, NPPA, ODC1,

RPS6KA1, SP110, SRPK3, TAF4

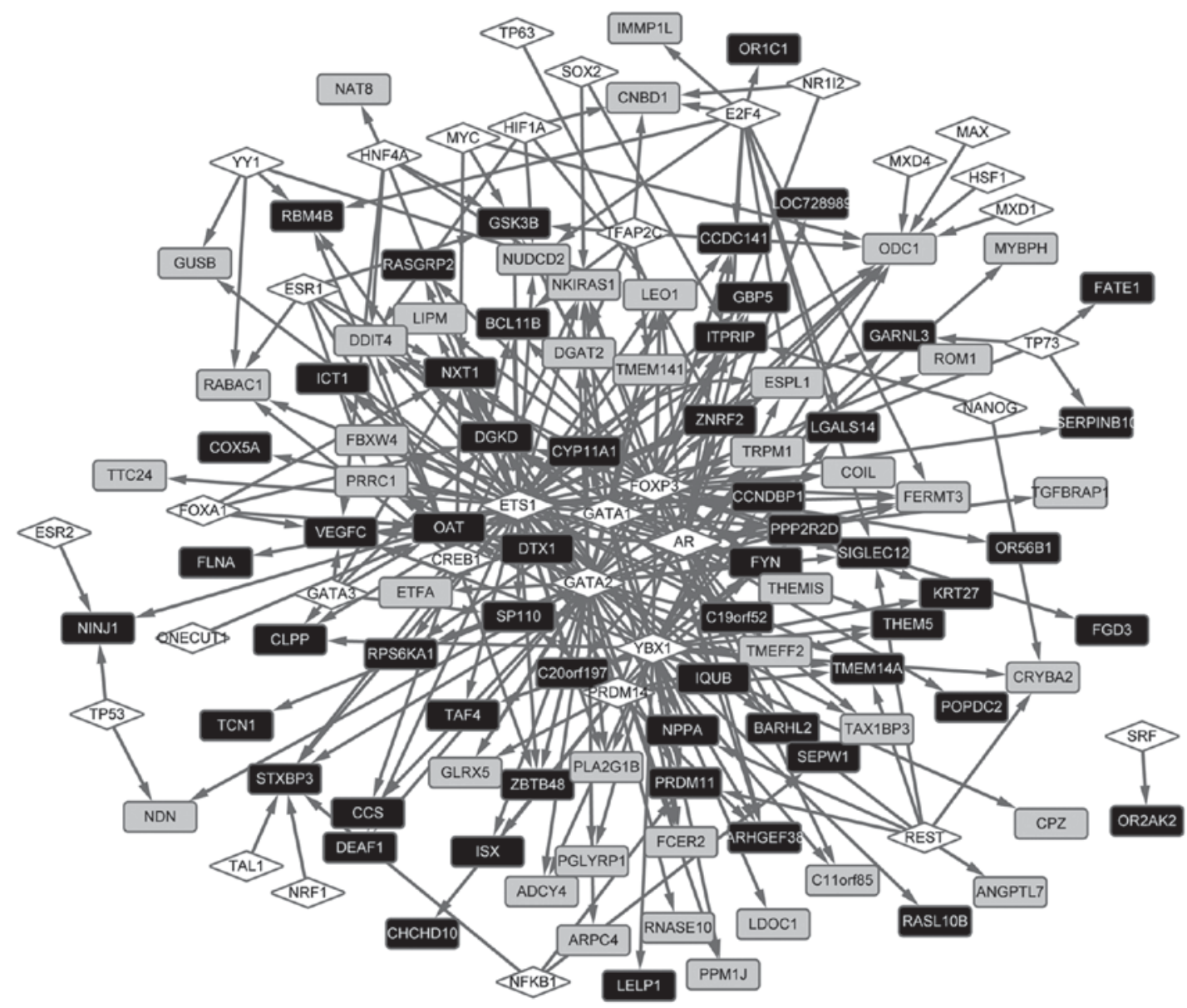

Figure 4. Transcriptional regulation network. The black nodes indicate upregulated genes. The gray nodes indicate the downregulated genes. The white diamonds represent transcription factors. The grey arrows indicate interactions between two nodes.

and has revealed the roles of immune cells and inflammatory mediators $(24,25)$. In addition, in the original analysis by Adigüzel et al, KEGG pathway analysis revealed that $14.6 \%$ of the DEGs identified were enriched in nodes of the immune system. Signals through the $\mathrm{T}$ cell receptor are shown to be significant for the initiation of $\mathrm{T}$ helper cell activation (26). Calvo et al (25) suggested that T cell involvement in the course of NP and modulation of the T lymphocyte phenotype may be one method for the management of NP. In accordance with the previous study, the present study found that DEGs were significantly enriched in the $\mathrm{T}$ cell receptor signaling pathway. Focal adhesion kinase is critical to cellular functions, including the migration, proliferation and survival of anchorage-dependent cells (27). Hua and Cabot (28) indicated that the immune system used the cell migration mechanisms not only to fight pathogens, but also to control pain within the injured issue. In accordance with the previous studies, the results of the present study showed that the DEGs were involved in the focal adhesion pathway. Taken together, the present study indicated that the focal adhesion and $\mathrm{T}$ cell receptor signaling pathways may be crucial for the development of NP following SCI. Genes, which were involved in these two pathways and active in lymphocytes may also be crucial in the molecular mechanisms of NP.

The protein encoded by $G S K 3 B$ is a serine-threonine kinase, which belongs to the $G S K$ subfamily (29). In previous 
years, GSK3 has been implicated in a number of roles in the immune system (30). Beurel et al (30) demonstrated that GSK3 is an important regulator of the balance between the production of pro- and anti-inflammatory cytokines in the peripheral and central nervous system. T cell differentiation, proliferation and survival were found to be affected by GSK3 (30). In the present study, GSK3B was a hub protein in the PPI network. In addition, it was found that $G S K 3 B$ was significantly linked to the focal adhesion and $\mathrm{T}$ cell receptor signaling pathways (Table II). As discussed above, genes involved in the focal adhesion and $\mathrm{T}$ cell receptor signaling pathways may be crucial for the development of NP following SCI. In this context, the present study indicated that active GSK3B in lymphocytes may be significant in the mechanisms underlying NP in patients with SCI, involved in the focal adhesion and $\mathrm{T}$ cell receptor signaling pathways. Furthermore, GSK3B may be a novel and effective pharmacological target for the treatment of NP following SCI, in accordance with a previous study, which showed that a GSK3-specific inhibitor, AR-A014418, produced marked antihyperalgesic effects in mice with NP (31). However, additional experimental verification is required to confirm this result.

$O D C 1$ encodes the rate-limiting enzyme of the polyamine biosynthesis pathway, which catalyzes ornithine to putrescine, a type of polyamine (32). Rivat et al (33) investigated the ability of a polyamine-deficient diet to prevent long-lasting pain hypersensitivity associated with tissue injury in rats, and found that the diet markedly reduced long-lasting hyperalgesia. In the present study, $O D C 1$ was associated with metabolic pathways and was concerned with the function of mitochondrial membrane. This indicated that $O D C 1$ may be crucial in PBMCs in the mechanisms of NP following SCI. By contrast, $O A T$ encodes the mitochondrial enzyme, ornithine aminotransferase, which is a key enzyme in the pathway converting arginine and ornithine into the major excitatory and inhibitory neurotransmitters, glutamate and gamma-aminobutyric acid (34). Studies have demonstrated that the balance of chloride ions, glutamate and gamma-aminobutyric acid distribution are disrupted following SCI, resulting in neuronal hyperexcitability and chronic NP (35). In addition, OAT and $O D C 1$ are involved in ornithine metabolism $(32,34)$. In accordance with the previous studies, the present study identified that $O A T$ and $O D C 1$ had interactions in the PPI network, and were regulated by a higher number of TFs. Taken together, the results suggested that the interactions of $O A T$ and $O D C 1$, which were involved in the metabolism process, may be key in the development and maintenance of chronic NP in patients with SCI. Further investigations are required to confirm this.

In conclusion, the data obtained in the present study demonstrated that gene expression profiles were altered in patients with SCI and NP, compared with the SCI patients without pain. The focal adhesion and $\mathrm{T}$ cell receptor signaling pathways may be crucial for the development of NP following SCI. GSK3B was found to be significantly involved in these two pathways, and may be a novel and effective pharmacological target for the treatment of NP. In addition, OAT may be key in its association with metabolic process in the development and maintenance of chronic NP following SCI, via interacting with $O D C 1$. Further investigations and experiments with additional patient cohorts, and delineation of the specific roles of these genes may lead to an improved understanding of the mechanisms underlying NP and the development of novel therapeutic options.

\section{Acknowledgements}

This study was supported by the Special Fund for Medical Service of Jilin Finance Department (grant no. SCZSY201507).

\section{References}

1. Cao HQ and Dong ED: An update on spinal cord injury research. Neurosci Bull 29: 94-102, 2013.

2. Finnerup NB and Baastrup C: Spinal cord injury pain: Mechanisms and management. Curr Pain Headache Rep 16: 207-216, 2012.

3. Mehta S, Orenczuk K, McIntyre A, Willems G, Wolfe DL, Hsieh JT, Short C, Loh E and Teasell RW; SCIRE Research Team: Neuropathic pain post spinal cord injury part 1: Systematic review of physical and behavioral treatment. Top Spinal Cord Inj Rehabil 19: 61-77, 2013.

4. Finnerup NB and Jensen TS: Spinal cord injury pain-mechanisms and treatment. Eur J Neurol 11: 73-82, 2004.

5. Von Schack D, Agostino MJ, Murray BS, Li Y, Reddy PS, Chen J, Choe SE, Strassle BW, Li C, Bates B, et al: Dynamic changes in the microRNA expression profile reveal multiple regulatory mechanisms in the spinal nerve ligation model of neuropathic pain. PLoS One 6: e17670, 2011.

6. Schomberg D, Miranpuri G, Duellman T, Crowell A, Vemuganti R and Resnick D: Spinal cord injury induced neuropathic pain: Molecular targets and therapeutic approaches. Metab Brain Dis 30: 645-658, 2015.

7. Saegusa $\mathrm{H}$ and Tanabe T: N-type voltage-dependent $\mathrm{Ca} 2+$ channel in non-excitable microglial cells in mice is involved in the pathophysiology of neuropathic pain. Biochem Biophys Res Commun 450: 142-147, 2014

8. Chen G, Park CK, Xie RG, Berta T, Nedergaard M and Ji RR: Connexin-43 induces chemokine release from spinal cord astrocytes to maintain late-phase neuropathic pain in mice. Brain 137: 2193-2209, 2014.

9. Nesic O, Lee J, Johnson KM, Ye Z, Xu GY, Unabia GC Wood TG, McAdoo DJ, Westlund KN, Hulsebosch CE and Regino Perez-Polo J: Transcriptional profiling of spinal cord injury-induced central neuropathic pain. J Neurochem 95: 998-1014, 2005.

10. Vicuña L, Strochlic DE, Latremoliere A, Bali KK, Simonetti M, Husainie D, Prokosch S, Riva P, Griffin RS, Njoo C, et al: The serine protease inhibitor Serpin A3N attenuates neuropathic pain by inhibiting $\mathrm{T}$ cell-derived leukocyte elastase. Nat Med 21: 518-523, 2015.

11. Sanchez-Palencia A, Gomez-Morales M, Gomez-Capilla JA, Pedraza V, Boyero L, Rosell R and Fárez-Vidal ME: Gene expression profiling reveals novel biomarkers in nonsmall cell lung cancer. Int J Cancer 129: 355-364, 2011.

12. Kim DS, Figueroa KW, Li KW, Boroujerdi A, Yolo T and Luo ZD: Profiling of dynamically changed gene expression in dorsal root ganglia post peripheral nerve injury and a critical role of injury-induced glial fibrillary acetic protein in maintenance of pain behaviors. Pain 143: 114-122, 2009.

13. Kim CF and Moalem-Taylor G: Interleukin-17 contributes to neuroinflammation and neuropathic pain following peripheral nerve injury in mice. J Pain 12: 370-383, 2011.

14. Barrett T, Wilhite SE, Ledoux P, Evangelista C, Kim IF, Tomashevsky M, Marshall KA, Phillippy KH, Sherman PM, Holko M, et al: NCBI GEO: Archive for functional genomics data sets-update. Nucleic Acids Res 41 (Database issue): D991-D995, 2013.

15. Altman NS: An introduction to kernel and nearest-neighbor nonparametric regression. The American Statistician 46: 175-185, 2012.

16. Hastie T, Tibshirani R, Narasimhan B and Chu G: impute: Imputation for microarray data. $\mathrm{R}$ package version 1, 2012.

17. López-Romero P, González MA, Callejas S, Dopazo A and Irizarry RA: Processing of Agilent microRNA array data. BMC Res Notes 3: 18, 2010.

18. Chen YA, Tripathi LP and Mizuguchi K: TargetMine, an integrated data warehouse for candidate gene prioritisation and target discovery. PLoS One 6: e17844, 2011. 
19. Szklarczyk D, Franceschini A, Kuhn M, Simonovic M, Roth A Minguez P, Doerks T, Stark M, Muller J, Bork P, et al: The STRING database in 2011: Functional interaction networks of proteins, globally integrated and scored. Nucleic Acids Res 39 (Database issue): D561-D568, 2011.

20. Shannon P, Markiel A, Ozier O, Baliga NS, Wang JT, Ramage D, Amin N, Schwikowski B and Ideker T: Cytoscape: A software environment for integrated models of biomolecular interaction networks. Genome Res 13: 2498-2504, 2003.

21. Bovolenta LA, Acencio ML and Lemke N: HTRIdb: An open-access database for experimentally verified human transcriptional regulation interactions. BMC Genomics 13: 405, 2012.

22. Wang JH, Zhao LF, Lin P, Su XR, Chen SJ, Huang LQ, Wang HF, Zhang H, Hu ZF, Yao KT and Huang ZX: GenCLiP 2.0: A web server for functional clustering of genes and construction of molecular networks based on free terms. Bioinformatics 30: 2534-2536, 2014.

23. Hagen EM and Rekand T: Management of neuropathic pain associated with spinal cord injury. Pain Ther 4: 51-65, 2015.

24. Moalem G and Tracey DJ: Immune and inflammatory mechanisms in neuropathic pain. Brain Res Rev 51: 240-264, 2006.

25. Calvo M, Dawes JM and Bennett DL: The role of the immune system in the generation of neuropathic pain. Lancet Neurol 11: 629-642, 2012 .

26. Huppa JB, Gleimer M, Sumen C and Davis MM: Continuous T cell receptor signaling required for synapse maintenance and full effector potential. Nat Immunol 4: 749-755, 2003.

27. Huang D, Khoe M, Befekadu M, Chung S, Takata Y, Ilic D and Bryer-Ash M: Focal adhesion kinase mediates cell survival via NF-kappaB and ERK signaling pathways. Am J Physiol Cell Physiol 292: C1339-C1352, 2007.
28. Hua S and Cabot PJ: Mechanisms of peripheral immunecell-mediated analgesia in inflammation: Clinical and therapeutic implications. Trends Pharmacol Sci 31: 427-433, 2010.

29. Galli C, Piemontese M, Lumetti S, Manfredi E, Macaluso G and Passeri G: GSK3b-inhibitor lithium chloride enhances activation of Wnt canonical signaling and osteoblast differentiation on hydrophilic titanium surfaces. Clin Oral Implants Res 24: 921-927, 2013.

30. Beurel E, Michalek SM and Jope RS: Innate and adaptive immune responses regulated by glycogen synthase kinase-3 (GSK3). Trends Immunol 31: 24-31, 2010.

31. Mazzardo-Martins L, Martins D, Stramosk J, Cidral-Filho F and Santos A: Glycogen synthase kinase 3-specific inhibitor AR-A014418 decreases neuropathic pain in mice: Evidence for the mechanisms of action. Neuroscience 226: 411-420, 2012.

32. Hogarty MD, Norris MD, Davis K, Liu X, Evageliou NF, Hayes CS, Pawel B, Guo R, Zhao H, Sekyere E, et al: ODC1 is a critical determinant of MYCN oncogenesis and a therapeutic target in neuroblastoma. Cancer Res 68: 9735-9745, 2008.

33. Rivat C, Richebé P, Laboureyras E, Laulin JP, Havouis R, Noble F, Moulinoux JP and Simonnet G: Polyamine deficient diet to relieve pain hypersensitivity. Pain 137: 125-137, 2008.

34. Bouché N, Lacombe Bt and Fromm H: GABA signaling: A conserved and ubiquitous mechanism. Trends Cell Biol 13: 607-610, 2003.

35. Gwak YS and Hulsebosch CE: GABA and central neuropathic pain following spinal cord injury. Neuropharmacology 60 : 799-808, 2011. 